\title{
Cuidadores de idosos: \\ um novo / velho trabalho
}

| 'Márcia Colamarco Ferreira Resende, 2Elizabeth Costa Dias |

Resumo: O texto tem como objetivo discutir as repercussôes do novo/velho trabalho dos cuidadores de idosos na sua saúde e o suporte que existe atualmente para eles. O estudo se fundamenta na referência do campo temático da Saúde do Trabalhador. Segundo a literatura, a ocupação de cuidador de idosos parece ser uma atividade exercida predominantemente dentro do setor informal de trabalho, por alguém da família e do sexo feminino, e tem trazido conseqüências na qualidade do cuidado ao idoso e na saúde do próprio cuidador, como dores lombares, depressão e hipertensão. A Política Nacional de Saúde da Pessoa Idosa é bastante avançada nas diretrizes para os cuidados com essa população e tem no cuidador familiar um parceiro para suas açôes. No entanto, estudos sobre as formas de trabalho dos cuidadores de idosos ainda são necessários para auxiliar na elaboração das ações das políticas públicas.

Palavras-chave: cuidadores, saúde do trabalhador, saúde ocupacional, saúde do idoso.

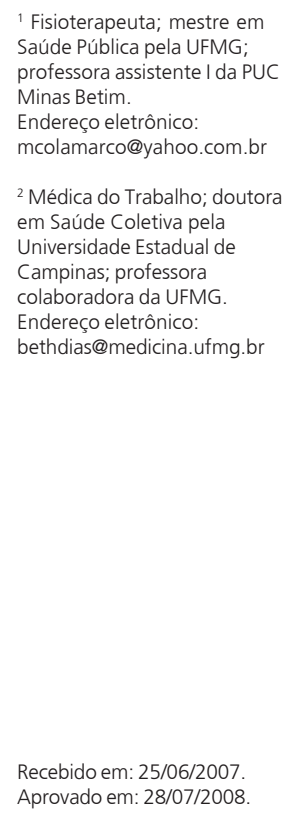




\section{Introdução}

Do ponto de vista filosófico, o cuidado é a essência da vida e é ele que permite "a revolução da ternura ao priorizar o social sobre o individual e ao orientar o desenvolvimento para a melhoria da qualidade de vida dos humanos e de outros organismos vivos. O cuidado faz surgir o ser humano complexo, sensível, solidário, cordial, e conectado com tudo e com todos no universo" (BOFF, 2004, p. 190).

Merhy (1998) discute a construção da prática de técnicas cuidadoras e aponta três tipos de ação de intervenção sobre o indivíduo. A primeira, o autor denomina "tecnologia dura", que são as intervenções assistenciais baseadas em ferramentasmáquinas que os profissionais de saúde utilizam, como raios-X e instrumentos para fazer exames de laboratórios. O segundo tipo é a "tecnologia leve-dura”, que associa as ferramentas anteriores, o acolhimento do paciente e os conhecimentos sobre a forma de saberes profissionais, bem estruturados, como a clínica do médico, o saber da fisioterapia, do psicólogo, etc. Por fim, o autor chama de "tecnologia leve" aqueles "momentos de falas, escutas e interpretações, no qual há a produção de uma acolhida ou não das intençôes dos pacientes; momentos de cumplicidades, nos quais há a produção de uma responsabilização em torno do problema que vai ser enfrentado; momentos de confiabilidade e esperança, nos quais se produzem relações de vínculo e aceitação" (MERHY, 1998, p. 106-107).

Diante da complexa configuração tecnológica do cuidado, defende-se a noção de que somente atuando em conformação com os três tipos de intervenção é que a dimensão cuidadora conseguirá apresentar resultados satisfatórios para os pacientes e seus familiares, para os sistemas de saúde e para os profissionais de saúde (MERHY, 1998). E só faz sentido cuidar de alguém quando este não conseguir desempenhar suas atividades de vida diária sem a ajuda de outras pessoas, sendo que muitas vezes esses cuidados podem ir além do âmbito domiciliar, no sentido de satisfazer as necessidades básicas do sujeito (consultas médicas, serviços bancários, lazer...).

Observando com atenção o rápido crescimento que a população idosa do Brasil teve nos últimos anos, visualiza-se também o aumento do número de idosos com doenças crônicas e múltiplas, que apresentam alguma forma de dependência e que demandam internações prolongadas e freqüentes IBGE, 2004; VERAS, 2003). Esta mudança de perfil de saúde da população idosa trouxe a necessidade de alterações nas formas de cuidado dessa população, e uma delas foi o retorno ao modelo de cuidados domiciliares. 
A Política Nacional de Saúde da Pessoa Idosa - PNSPI(BRASIL, 2006), que fundamenta as ações do poder público do setor saúde na atenção integral à população idosa do Brasil, incorpora o cuidado familiar e considera este modelo fundamental nos cuidados à saúde desse grupo populacional, configurando a junção das três dimensões cuidadoras. Nesse contexto, o cuidador familiar de idosos retorna como parte importante das ações de manutenção da autonomia, integração e participação do idoso na sociedade, além de ser determinante para o sucesso das diretrizes das políticas públicas. O incentivo ao retorno do modelo de cuidados domiciliares teve como principal finalidade baratear os altos custos advindos das internações dessa população e a melhora da qualidade de vida dos idosos. Mas a dificuldade das políticas públicas para acompanhar o rápido crescimento dessa população no país trouxe como conseqüência um enfoque distorcido da responsabilidade sobre as incapacidades ou as doenças crônicas do idoso, que foram assumidas por seus familiares como um problema individual e/ou familiar por causa da ausência, ou precariedade, do suporte do Estado (LEAL, 2000).

Apesar de o ramo de conhecimento filosófico levantar pontos positivos importantes nas açôes de cuidado, como a formação de um ser social e solidário, a realidade dos cuidadores de idosos parece ainda estar longe dessa idealização. Dentre os diversos obstáculos que se apresentam diante da assistência à saúde do país (financeiro, político, organizacional), a qualidade da atenção prestada também ganha destaque (AYRES, 2001). Segundo Deslandes e Ayres (2005), a qualidade do cuidado envolve o emprego de tecnologias, saberes, recursos adequados e todos esses fatores em um "contexto singular: o do encontro entre quem sofre, sejam indivíduos ou populações, e aqueles que se dedicam a mitigar este sofrimento, profissionais de saúde, gestores ou técnicos". Diversas especialidades vêm estudando os impactos do "encontro" sobre a saúde dos cuidadores familiares de idosos, sinalizando uma possível relação entre a atividade exercida na privacidade dos lares e o processo de saúde-adoecimento desse contingente de cuidadores (NAKATANI et al., 2003).

A relação existente entre o trabalho e a saúde dos indivíduos é constatada desde a Antiguidade, e vem sendo analisada pelo campo da Saúde do Trabalhador. Com o avanço da teoria da "determinação social das doenças" no processo de saúdeadoecimento das populaçóes, o trabalho passa a ter papel central nesse processo, uma vez entendido como organizador da vida social (MENDES; DIAS, 1991). 
Além de o trabalho ser uma atividade que produz bens materiais e que transforma o ambiente a sua volta, é também uma das atividades pela qual o homem produz a si mesmo. Segundo Lima (1998, p. 172), “o homem e o trabalho constituem os dois termos de uma relação de determinação reflexiva, na qual um não existe sem o outro e na qual um se define pelo outro. Desde seus primórdios, portanto, a forma como o trabalho é realizado depende e determina o que o homem é".

Segundo o conceito de trabalhador presente na Política Nacional de Saúde do Trabalhador (BRASIL, 2004), além dos indivíduos remunerados, inseridos no setor formal ou informal de trabalho, também são considerados trabalhadores aqueles indivíduos que, como os cuidadores familiares de idosos, realizam funçôes não remuneradas que fazem parte das atividades econômicas do lar.

É a partir da perspectiva da Saúde do Trabalhador que o presente estudo ${ }^{1}$ tem o objetivo de discutir as repercussões do novo/velho trabalho dos cuidadores de idosos na saúde dessa população e o suporte que existe atualmente para eles, dentro da produção científica dos últimos dez anos, tornando públicas as necessidades e dificuldades desse grupo de trabalhadores domiciliares.

\section{Metodologia}

Para a abordagem deste tema, foi realizada busca de artigos em periódicos indexados produzidos na última década, com particular ênfase nas publicações da área de Saúde Coletiva. As fontes consultadas foram o site do Scientific Electronic Library On-line - SciELO, organizado pela Fundação de Amparo à Pesquisa do Estado de São Paulo (Fapesp); as bases de dados Science Direct, Lilacs e MedLine/Pubmed, utilizando os seguintes descritores: cuidadores, idosos, caregiver, elderly, ageing, saúde do trabalhador, worker's health e a combinação deles. A busca permitiu selecionar textos em português, inglês e espanhol que descreveram a condição dos cuidadores e investigaram a relação entre o trabalho e a saúde desses indivíduos. Além disso, foi realizada busca sistemática sobre o tema nos sites do Ministério da Saúde e do Ministério do Trabalho e do Emprego, disponíveis em www.saude.gov.br e www.mte.gov.br, respectivamente. 


\section{Envelhecimento humano: um desafio para a saúde pública}

Para auxiliar no entendimento do tema, inicialmente faz-se necessário compreender as transformações que vêm ocorrendo com a população idosa do país, sua relação com o retorno ao modelo de cuidados domiciliares e a atual situação dos seus cuidadores.

Atualmente, a população idosa do Brasil representa um contingente de quase 15 milhōes de pessoas com 60 anos ou mais de idade (8,6\% da população brasileira), contra cerca de 11 milhōes em 1991 (7,3\% da população brasileira) (BGE, 2000). $\mathrm{O}$ aumento do número relativo e absoluto de idosos vem acompanhado de mudanças significativas no perfil epidemiológico dessa população. Nos últimos 40 anos, o Brasil passou de um perfil de saúde caracterizado pelas doenças infecto-contagiosas para um em que as enfermidades crônicas não-transmissíveis são predominantes (VERAS, 2003; LIMA-COSTA; BARRETO; GIATTI, 2003).

Ao incremento da idade e das doenças crônico-degenerativas podem se seguir seqüelas que comprometem a independência da população idosa. Lima-Costa, Barreto e Giatti (2003), em estudo com 28.943 idosos incluídos na amostra da PNAD de 1998, mostraram que 69,0\% dos idosos relataram ter pelo menos uma doença crônica, sendo esta proporção maior entre mulheres $(74,5 \%)$ do que entre os homens $(62,2 \%)$. Cerca de $15 \%$ dos participantes relataram ter dificuldades para alimentar-se, tomar banho ou ir ao banheiro. A impossibilidade para abaixarse, ajoelhar-se ou curvar-se foi relatada por cerca de 3\% dos homens e 6\% das mulheres. Além disso, a prevalência de incapacidade para realizar essas atividades aumentou com a idade em ambos os sexos.

Rosa et al. (2003) entrevistaram 964 idosos do município de São Paulo e encontraram um efeito dose-resposta entre a dependência moderada/grave e a faixa etária. Nos indivíduos entre 65 a 69 anos, a chance foi de aproximadamente 1,9 vezes maior, aumentando gradativamente até cerca de 36 vezes entre os de mais de 80 anos. O gênero também foi fortemente associado à ocorrência da dependência, sendo mais de duas vezes maior a chance para as mulheres em relação aos homens.

As transformações no perfil demográfico e epidemiológico da população idosa trazem grande impacto ao Sistema Público de Saúde do país, caracterizando um problema de Saúde Pública em decorrência da alta prevalência de doenças crônicodegenerativas e incapacitantes presentes nessa população. 
O Sistema Único de Saúde (SUS) parece ter que investir cada vez mais nas internações hospitalares de idosos. No ano de 1996, ocorreram 10,2 milhões de internaçôes hospitalares do tipo I pelo SUS e 15,8\% dessas internações ocorreram com pessoas acima dos 60 anos de idade, representando $22 \%$ do gasto com todas as internações hospitalares. No mesmo ano, a razão entre a proporção de gastos e a proporção de idosos na população total foi de 2,9 , sendo que esta razão aumentou significativamente com a idade, mostrando que o custo de hospitalização consumido por habitante foi mais alto para a faixa etária acima dos 60 anos (2000). Em 2001 esses números aumentaram. Os idosos representavam $14 \%$ da população adulta e contribuíram com $33,5 \%$ das internações hospitalares dessa população no SUS e $37,7 \%$ dos recursos pagos pelas mesmas (2004).

Esses dados demonstram que, consequentemente, a rápida mudança dos perfis de saúde do país, ocorre também o aumento do número de idosos potencialmente dependentes, que necessitam de cuidados especiais diariamente e consumidores de uma grande parcela dos serviços públicos de saúde. Uma das alternativas de cuidado para essa população são os asilos, casas de repouso e similares, mas a qualidade de vida dos idosos dentro dessas instituições está sendo discutida até em países onde estes serviços alcançaram níveis altamente sofisticados de conforto e eficiência. $\mathrm{O}$ custo desse modelo, as dificuldades de sua manutenção e o afastamento do idoso do ambiente familiar, tornam necessárias medidas alternativas que sejam mais resolutivas e menos onerosas (ROSA et al., 2003; KARSH, 2003).

A prática de cuidados no domicílio, por familiares dos idosos, retorna na atualidade como uma alternativa freqüente na atenção à população idosa, pois traz como ponto positivo o fato de que este suporte (amor, afeição, preocupação e assistência) tende a reduzir os efeitos negativos do estresse na saúde mental do idoso. Entretanto, podem existir resultados negativos desse suporte social em função da excessiva assistência ou dependência do idoso, em relação a poucas pessoas que possam ajudar (RAMOS et al., 2002).

A necessidade de cuidadores entre os idosos foi avaliada em uma pesquisa realizada na comunidade de Bambuí, MG (GIACOMIN et al., 2005). As autoras relataram que dos 1.606 idosos participantes da pesquisa, 23\% necessitavam de cuidador, sendo essa necessidade associada de forma estatisticamente significante com a faixa etária acima de 70 anos, ser solteiro, morar sozinho, o consumo regular de cinco ou mais drinques de bebida alcoólica em algum período da vida 
e história médica de hipertensão arterial (estágio 2), dentre outros. Além disso, verificou-se também que, em caso de adoecimento do idoso, a principal fonte de cuidados é o cônjuge ou a filha.

Outro fato que chama a atenção para o grande número de cuidadores familiares que vem surgindo nos últimos anos é a afirmação de Neno (2004), de que pelo menos um terço da população idosa pode ter a expectativa de ser cuidador de outro idoso durante a sua aposentadoria. Mas torna-se importante ressaltar que, segundo o autor, esses dados podem, ainda, subestimar o quadro real, porque muitos cônjuges não reconhecem a atividade do cuidado como uma ocupação ou trabalho e consideram que cuidar do marido/esposa é apenas mais uma responsabilidade matrimonial, mantendo suas queixas e dificuldades restritas ao lar.

A prática de cuidados no domićlío permaneceu por anos sendo pouco utilizada, em virtude do incentivo ao modelo hospitalocêntrico, e ressurge nos últimos anos como possível resposta às demandas da rápida transição demográfica e epidemiológica, e da elevação dos custos assistenciais à saúde dos idosos. Mas esta modalidade não pode ter como principais objetivos baratear custos ou transferir responsabilidades; ela deve também trazer a público todas as dificuldades experimentadas na relação cuidador/paciente que acontecem na privacidade dos lares e tentar dividir com o Estado a responsabilidade do cuidado com idoso dependente (LEAL, 2000; SILVESTRE; NETO, 2003). No entanto, esta parceria ainda é um grande desafio, pois apesar de a PNSPI (BRASIL, 2006) afirmar que o Estado tem um papel importante na saúde dos idosos capaz de otimizar o suporte familiar nos três níveis de gestão do SUS, sem transferir responsabilidades, a grande maioria da população ainda não usufrui do suporte determinado nas políticas públicas.

\section{Quem são os cuidadores familiares de idosos?}

Alguns estudos apontam a ocupação de cuidador de idosos como uma atividade exercida predominantemente dentro do setor informal de trabalho, por alguém da família e do sexo feminino (SILVA, 2004; BREWER, 2001; GARRIDO; MENEZES, 2004). No estudo desenvolvido por Karsch (2003), 92,9\% dos cuidadores entrevistados eram do sexo feminino, sendo que a maior parte era formada de esposas $(44,1 \%)$, seguidas pelas filhas (31,3\%). A faixa etária encontrada nessa população era a seguinte: $59 \%$ dos cuidadores estavam acima de 50 anos e $41 \%$ tinham mais de 60 anos. Além disso, os dados também mostraram que 
$39,3 \%$ de cuidadores, entre 60 e 80 anos, cuidavam de $62,5 \%$ de pacientes da mesma faixa etária, o que mostra que pessoas idosas estão cuidando de idosos.

Kuuppelomaki et.al. (2004) avaliaram 290 cuidadores de idosos de três cidades finlandesas e encontraram resultados parecidos com o do estudo descrito anteriormente. Segundo esses autores, 68\% dos cuidadores entrevistados eram mulheres e destas, $65 \%$ eram esposas e $17 \%$ eram filhas dos idosos. Quanto à idade, $22 \%$ dos cuidadores participantes tinham idade abaixo dos 50 anos, $22 \%$ entre 50 e 65 anos, 33\% estavam entre 66 e 75 anos e 23\% tinham idade superior a 76 anos.

É evidente que a determinação das funções de cuidador não está biologicamente relacionada ao sexo feminino, embora a função reprodutiva da mulher pareça influenciar nas forças psicossociais que moldam as práticas de cuidado. No entanto, também é provável que a observação e imitação prematura dos comportamentos cuidadores dentro da família (p.ex., a filha ajuda a mãe no cuidado da casa) desempenhem papel importante na reprodução dos cuidados femininos. Na sua maioria, a escolha do cuidador entre os gêneros é feita segundo a preferência da família, e não baseada nas habilidades pessoais ou experiência profissional prévia de quem vai cuidar (BREWER, 2001).

\section{O processo de saúde $\leftrightarrow$ doença dos cuidadores familiares de idosos}

\section{A saúde relacionada ao trabalho}

Até alguns anos atrás, os estudos em Saúde do Trabalhador tinham seu foco voltado para os impactos negativos do trabalho sobre a saúde dos indivíduos e das coletividades de trabalhadores. No entanto, recentemente, os aspectos positivos também começaram a receber atenção dos estudiosos no assunto, que estão identificando possíveis associações entre satisfação no trabalho e a saúde dos trabalhadores (MARTINEZ et al., 2004).

A satisfação no trabalho pode ser considerada como um fenômeno complexo, por se tratar de um estado subjetivo, que varia segundo as expectativas, valores e desejos de cada indivíduo, do mesmo indivíduo em diferentes momentos e das circunstâncias ambientais que os envolvem. Ela pode afetar a saúde física e mental do trabalhador, interferindo em seu comportamento profissional e/ou social. Como já foi dito, o trabalho tem papel importante na determinação do ser humano enquanto indivíduo social, e pode ser visto como um dos componentes da felicidade 
humana, na qual "a felicidade no trabalho é tida como resultante da satisfação de necessidades psicossociais, do sentimento de prazer e do sentido de contribuição no exercício da atividade profissional" (MARTINEZ et al., 2004).

Alguns autores têm demonstrado que existe grande satisfação por parte dos cuidadores de idosos dependentes ao realizar sua função cuidadora (KUUPPELOMÄKI et al., 2004, LOPÉZ; LOPÉZ-ARRIETA; CRESPO, 2005). Nestes estudos, a satisfação experimentada pelos cuidadores foi relacionada a uma boa relação afetiva prévia com o idoso, a manutenção dos momentos de lazer do cuidador, a sensação de ser útil e importante perante a família e a sociedade, e a não-imposição, pela família, do dever de cuidar (livre escolha).

Lopéz, Lopéz-Arrieta e Crespo (2005) afirmam que os aspectos positivos foram encontrados mesmo em situaçôes pouco favoráveis, que incluíam pacientes com graus avançados da doença de Alzheimer, insuficiência respiratória grave ou imobilidade, devido à capacidade do cuidador em superar estes problemas e/ou em manter uma boa relação afetiva com o idoso.

A força de associação entre a satisfação com o trabalho e a saúde dos trabalhadores parece persistir independentemente de características sóciodemográficas e culturais, o que evidencia a importância dos fatores psicossociais no trabalho em sua relação com a saúde nos aspectos de saúde mental e capacidade para o trabalho (MARTINEZ et al., 2004).

\section{$\mathrm{O}$ adoecimento relacionado ao trabalho}

A sociedade pode influenciar e definir o papel de cuidar à mulher, que normalmente tem filhos, marido, atividades domésticas além de muitas vezes trabalhar fora de casa. Segundo Giacomin et al. (2005), alguns fatores podem permear as dificuldades no cuidado cotidiano e colaborar para aumentar a sobrecarga de esposas que cuidam dos maridos. Dentre eles, a condição de saúde e as características de quem é cuidado, as limitações de quem cuida - muitas vezes relacionadas ao próprio processo de envelhecimento e adoecimento da cuidadora -, algumas situações de penúria social, experimentadas ao longo da vida, e a ausência de suporte institucional que poderiam facilitar o cuidado no domicílio.

Esta sobrecarga de papéis tem trazido conseqüências na qualidade do cuidado ao idoso e na saúde do próprio cuidador, mostrando que o corpo humano deu importantes sinais de desadaptação ao formato deste novo/velho trabalho. Alguns 
problemas de saúde que podem acometer os cuidadores são as dores lombares, depressão, artrite e a hipertensão arterial (NAKATANI et al., 2003; KARSH, 2003). A alta porcentagem de problemas de coluna parece estar intimamente relacionada com as atividades diárias de cuidados com os idosos, que envolvem o uso da força muscular e, muitas vezes, posturas inadequadas.

Investigar a relação da atividade dos cuidadores de idosos e a depressão que muitos deles apresentam tem despertado o interesse de vários pesquisadores há algum tempo. Segundo Matsuu et al. (2000), a alta prevalência de depressão entre os cuidadores de idosos pode estar associada ao tempo despendido com os cuidados ao idoso. Aqueles cuidadores que atendem aos idosos durante vários dias do mês e por várias horas no dia diminuem seu tempo para visitar amigos e/ou relaxar, e têm maiores chances de apresentar episódios de depressão. Beeson (2003) afirma, em seu estudo sobre solidão e depressão entre casais cuidadores e nãocuidadores, que a solidão é a única variável preditiva para depressão entre cuidadores de pacientes portadores da doença de Alzheimer. A importância do suporte familiar e da religiosidade sobre os episódios de depressão ocorridos entre os cuidadores de idosos foi estudada por Zunzunegui et al. (1999), e esses fatores foram associados à preservação da saúde mental dos cuidadores.

Pode-se ainda dizer que mudanças na demanda de cuidados por parte dos idosos ao longo do tempo podem influenciar os sintomas depressivos dos cuidadores. Um aumento nessa demanda resulta em aumento dos sintomas depressivos, ao passo que uma diminuição da demanda de cuidados pode diminuir tais sintomas nos cuidadores familiares de idosos, mostrando íntima relação entre a atividade desenvolvida pelo cuidador e a sua saúde NIEBOER et al., 1998).

Quanto à hipertensão arterial (HA), sabe-se que hábitos saudáveis de vida e a herança genética são importantes fatores de risco, mas a influência dos fatores psicossociais ainda não é muito clara. Através de estudos longitudinais, alguns autores verificaram que o estresse crônico promovido no cuidado de pacientes portadores da doença de Alzheimer também pode aumentar o risco de HA ou piorar a condição dos indivíduos que já tinham este diagnóstico. Esta afirmativa foi significativa para os primeiros três anos do estudo e diminuiu muito após o quarto ano, provavelmente por causa da morte do cônjuge ou troca de cuidadores (SHAW et al., 1999 e 2003). 


\section{Quem cuida dos cuidadores?}

O que existe atualmente são experiências isoladas, que tentam fornecer suporte a determinado grupo de cuidadores de idosos, por um tempo predeterminado e desarticuladas do sistema público. Em estudo realizado no Centro de SaúdeEscola da Faculdade de Medicina de Botucatu (UNESP), Cerqueira e Oliveira (2005) constataram que após um programa de apoio aos cuidadores de idosos, com 10 a 12 reuniōes coordenadas por duas psicólogas, estes indivíduos apresentaram mudanças favoráveis na expressão dos sentimentos e a retomada das atividades de lazer.

No Reino Unido, Mant et al. (2000) realizaram um estudo com os pacientes que deram entrada em alguns hospitais de Oxford, entre agosto de 1995 e fevereiro de 1998, com o diagnóstico de acidente vascular cerebral (AVC). O objetivo era avaliar o impacto do suporte familiar nos pacientes com AVC e nos seus cuidadores. Os autores mostraram que o suporte familiar não teve um benefício significativo para os pacientes, mas, assim como no estudo de Botucatu, foi significativamente benéfico em relação às atividades sociais e a qualidade de vida dos cuidadores, embora o mecanismo que levou a estes benefícios psicossociais não tenha ficado claro para os autores.

Em Samoa, um grupo de enfermeiras realizou um trabalho diferenciado com a população do país, que não ultrapassa os $\mathbf{1 7 5 . 0 0 0}$ habitantes. Foram elaboradas oficinas educativas para cuidadores de idosos, com o objetivo de desenvolver um programa de educação barato direcionado para eles, de determinar o impacto das oficinas sobre os cuidadores e sobre os cuidados com os idosos, e auxiliar na determinação de políticas específicas para os idosos de Samoa (MULATILO et al., 2000). Depois de mais de duas semanas de oficinas e 540 cuidadores participantes, verificou-se que as informaçōes foram efetivas para os cuidadores, que os cuidados com os idosos melhoraram, que a comunidade se tornou mais sensível às questôes relacionadas ao envelhecimento, além de ter sido criado um grupo interdisciplinar para discutir e elaborar políticas governamentais de apoio aos idosos.

Infelizmente, no Brasil, a Política Nacional de Saúde da Pessoa Idosa afirma que "embora a legislação brasileira relativa aos cuidados da população idosa seja bastante avançada, a prática ainda é insatisfatória” (BRASIL, 2006). Essa afirmativa acentua o desafio de se entender/compreender melhor as condições de trabalho dos cuidadores de idosos e as suas repercussões sobre o processo de saúde-adoecimento 
destes indivíduos, para que o Estado possa desenvolver ferramentas direcionadas para um melhor suporte ao trabalho dessa população.

\section{Considerações finais}

Diversos estudos têm discutido a questão dos cuidadores de idosos, no entanto as exigências concretas necessárias ao cuidado de um idoso dependente dentro do domicílio, ainda são obscuras, tornando necessárias pesquisas sobre os elementos de sobrecarga desta atividade.

No Brasil, infelizmente o sistema de saúde público ainda está se preparando para atender a crescente demanda de idosos e a de seus familiares. Mas no momento em que a PNSPI considera os cuidadores de idosos parceiros das equipes de saúde na assistência aos idosos dependentes, ela se torna responsável por fornecer condições dignas de trabalho para esses "agentes de saúde". A constatação de que o processo de saúde e doença dos cuidadores familiares de idosos tem relação com suas atividades cuidadoras, leva crer que o suporte à essa população deve ir além das técnicas cuidadoras com os idosos, e abordar também as formas de organização desse trabalho.

\section{Referências}

AYRES, J.R C.M. Sujeito, intersubjetividade e práticas de saúde. Ciência \& Saúde Coletiva, v. 6, n. 1, p. 63-72, 2001.

BEESON, R.A. Loneliness and Depression in Spousal Caregivers of Those With Alzheimer's Disease Versus Non-Caregiving Spouses. Archives of Psychiatric Nursing, v. XVII, n. 3, p. 135143, June 2003.

BOFF, L. Conclusão. In: Saber cuidar: ética do humano - compaixão pela terra. 11 ed.

Petrópolis: Vozes, 2004.

BRASIL. Ministério da Saúde. Portaria nº 800, de novembro de 2004. Política Nacional de Saúde do Trabalhador para o SUS.

BRASIL. Ministério da Saúde. Portaria no 2.528, de outubro de 2006. Política Nacional de Saúde da Pessoa Idosa.

BREWER, L. Gender socialization and the cultural construction of elder caregivers. Journal of Aging Studies, v. 15, n. 20, p. 217-235, 2001.

CERQUEIRA, A.T.A.R.; OLIVEIRA, N.I.L. Programa de apoio a cuidadores: uma ação terapêutica 
e preventiva na atenção à saúde dos idosos. Psicol. USP. São Paulo, v. 13, n. 1, 2002. Disponível em <www.scielo.com.br> Acesso em: 23 jul. 2005.

DESLANDES, S.F.; AYRES, J.R.C.M. Humanização e cuidado em saúde. Ciência \& Saúde Coletiva. Rio de Janeiro, v. 10, n. 3, p. 510-510, jul./set. 2005.

GARRIDO, R.; MENEZES, P.R. Impacto em cuidadores de idosos com demência atendidos em um serviço psicogeriátrico. Revista de Saúde Pública, v. 38, n. 6, p. 835-41, 2004.

GIACOMIN, K.C.; UCHÔA, E.; FIRMO, J.O.A. et al. Projeto Bambuí: um estudo de base populacional da prevalência e dos fatores associados à necessidade de cuidador entre idosos. Cad. Saúde Pública. Rio de Janeiro, v. 21, n. 1, p. 80-91, jan-fev. 2005.

INSTITUTO BRASILEIRO DE GEOGRAFIA E ESTATÍSTICA. Perfil dos idosos responsáveis pelos domicílios no Brasil, Rio de Janeiro, 2000. Disponível em <http://www.ibge.gov.br/home/ estatistica/populacao/perfilidoso/ perfidosos2000.pdf>. Acesso em: 30 out. 2004.

KARSCH, U.M. Idosos dependentes: famílias e cuidadores. Cad. Saúde Pública. Rio de Janeiro, v. 19, n. 3, p. 861-866, mai-jun. 2003.

KUUPPELOMÄKI, M.; SASAKI, A.; YAMADA, K. et al. Family carers for older relatives: sources of satisfaction and related factors in Finland. International Journal of Nursing Studies, v. 41, p. 497-505, 2004.

LEAL, M.G.S. O desafio da longevidade e o suporte ao cuidador. Revista da Terceira Idade. Publicação do SESC, Ano XI, n. 20, ago. 2000.

LIMA, F.P.A. Noções de organização do trabalho. In: OLIVEIRA, C.R. Manual prático de LERLesôes por Esforços Repetitivos. Belo Horizonte: Health, 1998.

LIMA-COSTA, M.F.; BARRETO, S.M.; GIATTI, L. Condições de saúde, capacidade funcional, uso de serviços de saúde e gastos com medicamentos da população idosa brasileira: um estudo descritivo baseado na Pesquisa Nacional por Amostra de Domicílios. Cad. Saúde Pública. Rio de Janeiro, v. 19, n. 3, p.735-743, mai-jun. 2003.

LIMA-COSTA, M.F.; PEIXOTO, S.V.; GIATTI, L. Tendências da mortalidade entre idosos brasileiros (1980 - 2000). Epidemiologia e Serviços de Saúde. Brasília, v. 13, n. 4, p. 217-228, outdez. 2004.

LIMA-COSTA, M.F.F.; GUERRA, H.L.; BARRETO, S.M. et al. Diagnóstico de saúde da população idosa brasileira: Um estudo da mortalidade e das internações hospitalares públicas. Informe Epidemiológico do SUS, v. 9, p. 23-41; 2000.

LOPÉZ, J.; LOPÉZ-ARRIETA, J.; CRESPO, M. Factors associated with the positive impact of caring for elderly and dependent relatives. Archives of Gerontology and Geriatrics, v. 41, p. 81-94, 2005.

MANT, J.; CARTER, J.; WADE, D.T.et al. Family support for stroke: A randomised controlled trial. The Lancet, Health \& Medical Complete, p. 808, Sept. 2, 2000. 
MARTINEZ, M.C.; PARAGUAY, A.I.B.B.; LATORRE, M.R.D.O. Relação entre satisfação com aspectos psicossociais e saúde dos trabalhadores. Revista de Saúde Pública, v. 38, n. 1, p. 55-61, 2004. MATSUU, K.; WASHIO, M.; ARAI, Y. et. al.Depression among caregivers of the frail elderly in urban Japan. Psychiatry and Clinical Neurosciense, v. 54, p. 553-557, 2000.

MENDES, R.; DIAS, E.C. Da medicina do trabalho à saúde do trabalhador. Revista de Saúde Pública, v. 25, p. 341-349; 1991.

MERHY, E.E. A perda da dimensão cuidadora na produção da saúde: uma discussão do modelo assistencial e da intervenção no seu modo de trabalhar a assistência. In. Sistema Único de Saúde em Belo Horizonte: reescrevendo o público. São Paulo: Xamã, 1998. p. 103-120.

MULATILO, M.; TAUPAU, T.; ENOKA, I. et al. Teaching families to be caregivers for the elderly, Nursing and Health Science, v. 2, p. 51-58, 2000.

NAKATANI, A. Y. K.; SOUTO, C.C.S.; PAULETTE, L. M. et al. Perfil dos cuidadores informais de idosos com déficit de autocuidado atendidos elo Programa de Saúde da Família. Revista Eletrônica de Enfermagem, v. 5, n. 1, 2003. Disponível em < http:/www.fen_ufg.br/revista>. Acesso em: 10 nov. 2004.

NENO, R. Spouse caregivers and the support they receive: a literature review. Nursing Older People, v. 16, n. 5, p. 14, jul-aug. 2004.

NIEBOER, A.P.; SCHULZ, R.; MATTHEWS, K. A. et al. Spousal caregivers' activity restriction and depression: a model for changes over time. Soc. Sci. Med, v. 47, n. 9, p. 1.361-1.371, 1998. PEIXOTO, S. V. et al. Custo das internações hospitalares entre idosos brasileiros no âmbito do Sistema Único de Saúde. Epidemiologia e Serviços de Saúde, Brasília, v. 13, n. 4, p. 239-246, 2004. RAMOS, M. P. P. Apoio social e saúde entre idosos. Sociologias. Porto Alegre, ano 4, n. 7, p. $156-$ 175, jan-jun. 2002.

ROSA, T.E.C.; BENÍCIO, M.H.D.; LATORRE, M.R.D.O. et al. Fatores determinantes da capacidade funcional entre idosos. Rev Saúde Pública, v. 37, n. 1, p. 40-8, 2003.

SHAW, W.S.; PATTERSON, T.L.; SEMPLE, S. J. et al. Emotional expressiveness, hostility and blood pressure in a longitudinal cohort of Alzheimer caregivers. Journal of Psychosomatic Research, v. 54, p. 293-302, 2003.

SHAW, W.S.; ZIEGLER, M. G. et al. Accelerated risk of hypertensive blood pressure recordings among Alzheimer caregivers. Journal of Psychosomatic Research, v. 46, n 3, p. 215-227, 1999.

SILVA, L.R. El cuidado en el hogar a los enfermos crónicos: un sistema de autoatención. Cad. Saúde Pública. Rio de Janeiro, v. 20, n. 2, p. 618-625, mar-abr. 2004.

SILVESTRE, J.A.; NETO, M.M.C. Abordagem do idoso em programas de saúde da família. Cad. Saúde Pública. Rio de Janeiro, v. 19, n. 3, p. 839-847, mai-jun. 2003. 
VERAS, R. Em busca de uma assistência adequada à saúde do idoso: revisão da literatura e aplicação de um instrumento de detecção precoce e de previsibilidade de agravos. Cad. Saúde Pública, Rio de Janeiro, v. 19, n. 3, p. 705-715, mai-jun. 2003.

ZUNZUNEGUI, M-V.; BÉLAND, F.; LLÁCER, A. et al. Family, religion, and depressive symptoms in caregivers of disabled elderly. J Epidemiol Community Health, v. 53, p.364-369, 1999.

\section{Nota}

${ }^{1}$ Este artigo faz parte de uma pesquisa mais ampla, intitulada As condições de trabalho e a saúde dos cuidadores de idosos do território de uma Unidade Básica de Saúde de Betim, MG, apresentada como dissertação ao Programa de Pós-Graduação em Saúde Pública da Universidade Federal de Minas Gerais, como requisito para a obtenção do título de Mestre em Saúde Pública/Saúde e Trabalho. Sua realização foi aprovada pelo Comitê de Ética em Pesquisa da UFMG, no processo ${ }^{\circ} \mathrm{CAAE}$ 0470.0.203.000-05. 


\section{Abstract}

\section{Elderly caregivers: a new/old work}

This paper aims to identify in literature the repercussions of the new/old work elderly caregivers concerning their health care and their current support. The study is based on the Workers' Health area. According to literature, the occupation of "elderly caregiver" seems to be an activity mainly developed in the informal sector, by some relative, usually women, and it has brought consequences for the quality of the elderly care and for the caregiver's health, causing low back pains, depression and hypertension. The National Health Politics of the Elderly is modern when it talks about the care for this population and the family caregiver is a partner. However, studies on this kind of work are still necessary to help making public policies.

> Key words: caregivers, occupational health, elderly's health. 